J. Dairy Sci. 98:3559-3567

http://dx.doi.org/10.3168/jds.2014-8548

(C) American Dairy Science Association ${ }^{\circledR}, 2015$.

\title{
Quantification of whey in fluid milk using confocal Raman microscopy and artificial neural network
}

\author{
Roney Alves da Rocha, ${ }^{* 1}$ Igor Moura Paiva, $†$ Virgílio Anjos, ${ }^{*}$ Marco Antônio Moreira Furtado,† \\ and Maria José Valenzuela Bell*1 \\ *Departamento de Física, Instituto de Ciências Exatas, and \\ †Departamento de Ciências Farmacêuticas, Faculdade de Farmácia, Universidade Federal de Juiz de Fora, Juiz de Fora, Minas Gerais, \\ 36036-900, Brazil
}

\section{ABSTRACT}

In this work, we assessed the use of confocal Raman microscopy and artificial neural network as a practical method to assess and quantify adulteration of fluid milk by addition of whey. Milk samples with added whey (from 0 to $100 \%$ ) were prepared, simulating different levels of fraudulent adulteration. All analyses were carried out by direct inspection at the light microscope after depositing drops from each sample on a microscope slide and drying them at room temperature. No pre- or posttreatment (e.g., sample preparation or spectral correction) was required in the analyses. Quantitative determination of adulteration was performed through a feed-forward artificial neural network (ANN). Different ANN configurations were evaluated based on their coefficient of determination $\left(\mathrm{R}^{2}\right)$ and root mean square error values, which were criteria for selecting the best predictor model. In the selected model, we observed that data from both training and validation subsets presented $\mathrm{R}^{2}>99.99 \%$, indicating that the combination of confocal Raman microscopy and ANN is a rapid, simple, and efficient method to quantify milk adulteration by whey. Because sample preparation and postprocessing of spectra were not required, the method has potential applications in health surveillance and food quality monitoring.

Key words: Raman spectroscopy, artificial neural network, milk adulteration, whey

\section{INTRODUCTION}

Milk has a rich and diversified composition and is an important source of food in the human diet worldwide (Kapila et al., 2013; Unluturk et al., 2013). Even though whey is a food rich in nutrients, with high nutritional

Received June 30, 2014.

Accepted January 6, 2015.

${ }^{1}$ Corresponding author: mjbell@fisica.ufjf.br value, it is 4 to 5 times cheaper than milk and continues to be a problem as a waste product in the cheese industry due to its high biological oxygen demand (Neelima et al., 2013). The addition of whey to milk constitutes a common fraud, because it results in an increase in volume without significantly changing the total protein content and without changing the sensorial quality.

Several methods have been used to detect and quantify whey in milk, many of which have focused on glycomacropeptide (GMP). Detection of sialic acid by colorimetric methods is another approach to determine this type of adulteration (Warren, 1959; Koning et al., 1966; Wolfschoon-Pombo and Pinto, 1985). Other methods involving chromatography (Kawakami et al., 1992; Olieman and Bedem, 1983), SDS-PAGE (Vilela, 1987; Galindo-Amaya et al., 2006), capillary electrophoresis (Recio et al., 2000), Western blot (Chávez et al., 2008), ELISA (Chávez et al., 2012), and fluorimetry (Neelima et al., 2012) have been applied to qualitative and quantitative analyses of GMP.

In spite of the benefits of these traditional methods, in most cases, time spent in sample preparation and analysis to obtain results is extensive (Jawaid et al., 2013; Giovannozzi et al., 2014), which means these methods cannot be applied routinely in the food industry and in sanitary vigilance laboratories.

Fast and nondestructive analytical methods with simple preprocessing (milk preparation) and postprocessing (date analysis) procedures are in great demand for many purposes. Because adulteration of fluid milk and milk powder with whey is prevalent in Brazil, the government has published official method to quantify this adulterant based on GMP analysis by chromatography after pretreatment with TCA (Brazil, 2006). However, in addition to being time consuming, the method has the drawback of interference from GMP obtained by proteolytic activity from psychotropic bacteria (Recio et al., 2000), especially among milk samples collected from farms with inadequate microbial quality or after extended refrigerated storage (Sorhauge and Stepaniak, 1997). 
Rapid approaches using chemometric methods have many advantages because sample preparation and wet laboratory procedures are not required. Borin et al. (2006) proposed the use of near-infrared (NIR) spectroscopy with a modified support vector machine for quantifying adulterants in milk powder, such as whey, starch, and sucrose. They showed that, for multivariate calibrations, nonlinear models are better than linear models. Mid-infrared spectra have also been used for detecting and quantifying synthetic milk (preparation of vegetable oils with detergents and urea), whey, urea, and hydrogen peroxide (Santos et al., 2013). Zhang et al. (2014) used NIR spectra and a modified support vector machine for identification of dextrin or starch with melamine, urea, or ammonium nitrate in fluid milk, but only levels of adulteration equal to or greater than $5 \%$ could be recognized.

Another promising technique is confocal Raman microscopy (Dieing and Hollricher, 2008). It is based on the same principles as Raman spectroscopy, but it uses an optical microscope to focus the laser in specific planes and spots along the sample (López-López and García-Ruiz, 2014). The Raman technique is primarily advantageous because each material has a unique and unequivocal spectrum (fingerprint). No sample preparation is required, analysis time is brief, and materials in different physical states and dimensions can be analyzed (McCreery, 2000; Dieing and Hollricher, 2008). Moreover, the operating cost of confocal Raman microscopy is relatively low, with no cost for chemical reagents, solvents, or vials.

Raman spectroscopy has been used for a wide variety of purposes, such as evaluating the partitioning of volatile compounds presented in milk fat globules (Zheng et al., 2013) and determining the addition of whey to milk powder (Almeida et al., 2011). Giovannozzi et al. (2014) developed surface-enhanced Raman scattering, exploring the selective linkage of gold nanoparticles to melamine, to rapidly detect this hazardous compound in milk.

Neural networks have been widely used in chemometrics to replace traditional multivariate calibration methods based on models of multiple linear regression because they are able to efficiently map and extract nonlinear, noisy, or incomplete relationships from the study data (Ramji et al., 2009; Chakraborty and Sahu, 2014). Artificial neural networks (ANN) come from artificial intelligence, a branch of computing science that tries to understand and model the behavior of the human brain (Haykin, 1999). Artificial neural networks use a set of parallel and symbolic processing algorithms and are implemented mainly for data prediction, grouping, and classification (Baughman and Liu, 1995).
A neural network is able to fit a mathematical model of algorithm input information (independent variables) and output information (dependent variables) present in a data set by establishing a functional relationship between them. This model fitting occurs iteratively and is called supervised training. Unsupervised training can be used especially in classification and clustering analysis. In mathematical terms, the neural network is similar to a directed graph. It is composed of nodes and edges, where the nodes are called "neurons" or "processing elements (PE)" and edges are called "synapses," analogous to biological systems. Neurons can be input or output information. The input layer is the name given to the input node set, and the output layer is the set of information of output nodes. The neurons between the input and output layers form the hidden layer and they do all the internal network processing and establish a functional relationship between the information coming to the input layer and leaving the network at the layer exit. Some neural networks have 2 or 3 hidden layers. In this work, we used supervised training with ANN formed by a single hidden layer. Full details of the design, construction, analysis, and neural network applications can be found in Haykin (1999).

This work proposes the use of confocal Raman microscopy and ANN to obtain an efficient predictor model that can quantify adulteration of milk by addition of whey across a wide range of adulteration levels. This methodology is a potential analytical tool for industry and food monitoring.

\section{MATERIALS AND METHODS}

The present study was carried out in Brazil, with milk from the region of Juiz de Fora, Minas Gerais state. Measurements were performed in the Laboratory of Spectroscopy of Materials from the Department of Physics, and in the Laboratory of Food Research from the Faculty of Pharmacy of the Federal University of Juiz de Fora.

\section{Materials}

Pasteurized milk samples (from a regional brand) were purchased from the dairy plant Marvim Produtos Agropecuários Ltda, located near Juiz de Fora. Percentage values of protein $(3.5 \%)$, fat $(3.0 \%)$, and carbohydrate $(5.0 \%)$ were indicated by the manufacturer.

Sweet whey was obtained on a laboratory scale by milk enzymatic coagulation using chymosin produced by Aspergillus niger var. awamori (coagulant HA-LA, Chr. Hansen, Valinhos, Brazil). Two milliliters of liquid coagulant was added to $5 \mathrm{~L}$ of milk. Incubation time 
was 40 min at $36^{\circ} \mathrm{C}$. Afterward, the curd was cut into cubes, stirred, and lightly pressed by hand. The mass was moved slowly and constantly for $30 \mathrm{~min}$. Afterward, whey was filtered using a sieve and then through a paper filter, where whey was collected. Finally, whey was incubated at $100^{\circ} \mathrm{C}$ for 5 min before using it in the preparation of simulated adulterated samples.

Intentional adulterations were prepared by adding sweet whey into milk, in different mixture percentages $[0,0.25,0.5,0.75,1,1.5,2,2.5,3,4,5,10,15, \ldots, 90$, $95,100 \%$ (i.e., $0 \%$ milk), wt/wt], simulating a total of 30 levels of adulteration. Mixtures were prepared in volumetric flasks and shaken thoroughly to guarantee their homogenization.

\section{Raman Instrumentation}

For confocal Raman microscopic observations, $1 \mu \mathrm{L}$ from each sample was pipetted onto a flat microscope slide in a circle of $2 \mathrm{~cm}$ diameter and naturally air-dried for $2 \mathrm{~h}$. Raman spectra were collected using a Horiba Jobin Yvon T64000 triple monochromator spectrometer (Horiba Jobin Yvon, Lille, France) with spectral resolution of $0.5675 \mathrm{~cm}^{-1}$. The micro-Raman system consisted of a $\mathrm{N}_{2}$-cooled charge-coupled device (CCD) detector and an Olympus $100 \times$ microscope objective (both from Horiba Jobin Yvon) with a 50- $\mu$ m confocal pinhole. The spectral region recorded was from 1,200 to $1,600 \mathrm{~cm}^{-1}$. Each sample (dried microdrop) was excited in 3 different microscopic fields (spots) using a single argon ion laser tuned to $514.5 \mathrm{~nm}$, so that final spectrum of each dried microdrop consisted of the average of 3 spectra. Measurements were carried out at room temperature. Some spike noises in Raman spectra due to cosmic rays that reach the CCD detector during data acquisition were removed before chemometric analyses. This noise is easily seen by visual inspection of the spectrum because the spikes produce typical very sharp and intense peaks that differ from all other peaks of the recorded spectrum. In general, spikes are removed automatically by the Raman driver software but can also be removed manually.

\section{Calibration with ANN}

Initially, to construct the predictor model, Raman spectra were discretized into wavelength intervals of approximately $0.57 \mathrm{~cm}^{-1}$, totaling 715 points. We applied no baseline correction, normalization, smoothing, or filtration of the Raman spectra. After discretization, the 90 spectra were used for both training and validation of the neural networks, characterized by an input layer, an output layer, and one hidden layer, as shown in Figure 1.

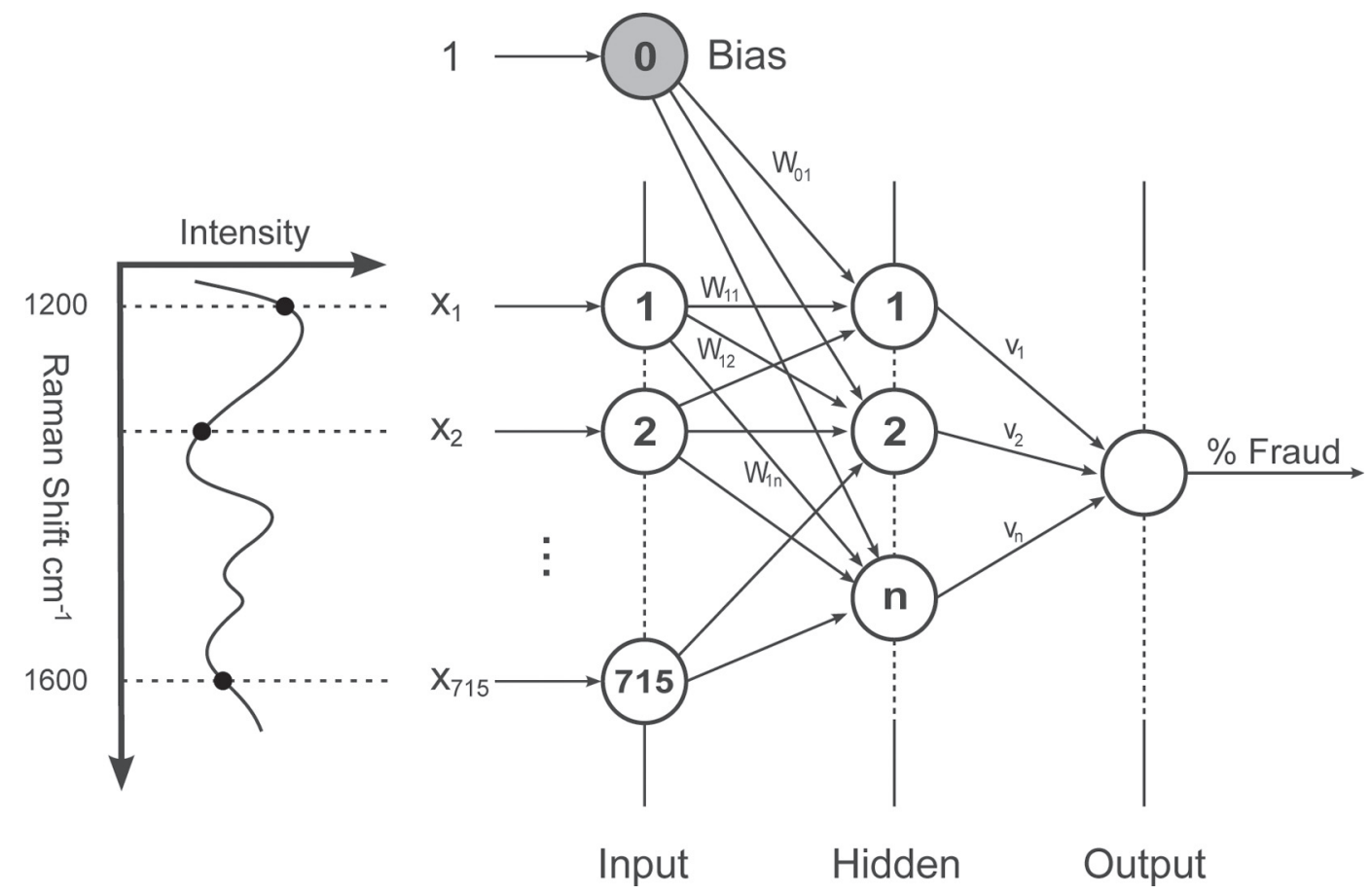

Figure 1. Diagram of the discretization of the spectra and neural network topology used in the calibration of the percentage of fraud by the addition of whey. $\mathrm{x}_{\mathrm{n}}$ ' $=$ input number that feeds the input layer, where $\mathrm{n}^{\prime}=1$ to $715 ; \mathrm{W}_{\mathrm{n}^{\prime} \mathrm{n}}=$ links that connect the input node layer with the hidden layer; $n^{\prime}=1$ to 715 and $n=5$ to 20 ; and $V_{n}=$ links that connect the hidden layer to the output layer. 
Table 1. Parameters used for training and validation of the artificial neural network

\begin{tabular}{ll}
\hline Parameter & Value \\
\hline Method & $\begin{array}{l}\text { Broyden-Fletcher-Goldfarb-Shanno } \\
\text { algorithm }\end{array}$ \\
Cross validation & k-fold method $(5 \text { groups })^{1}$ \\
Hidden nodes & 5 to 20 \\
Overfit penalty & 0.01 \\
Number of tours & 4 \\
Maximum iterations & 200 \\
Convergence criterion & 0.00001 \\
Transfer function & $\operatorname{Tanh}()^{2}$
\end{tabular}

${ }^{1}$ Cross validation method, where the sample is divided into $\mathrm{k}$ subsamples where 1 is retained to test the statistical model and the other $\mathrm{k}-1$ are used as training data.

${ }^{2}$ Hyperbolic tangent () .

The neural module from the statistical package SAS JMP Pro (version 11; SAS Institute Inc., Cary, NC) was used for training and validation of the networks. Training data refers to the data set used to effectively adjust the predictor model to experimental data, whereas validation data refers to another set of data previously reserved and is not used to adjust the predictor model but to check if the model is properly tuned. The predictor model should be able to correctly estimate the other values, different from those used during adjustment. The validation data are used only when the predictor model is ready and are different from those used for training data. In general, $75 \%$ of all experimental data are reserved for training, and the remaining $25 \%$ are used for validation.

The partial least squares (PLS) module from the same software was used to perform a test using multivariate regression by partial least squares. Only one node was used in the output layer, corresponding to the adulteration percentage. The hidden layer consisted of several nodes (between 5 and 20). The feed forward topology was implemented in all configurations. Values of $\mathrm{R}^{2}$ and root mean square error (RMSE) from training and validation data were used as selection criteria for the best predictor model. Analyses were carried out using a micro PC Intel i5, with 6 GB of RAM. Parameters tested for construction of networks are specified in Table 1.

\section{RESULTS AND DISCUSSION}

Figure 2 shows Raman spectra of 5 selected samples of adulterated milk in the range from 1,200 to 1,600 $\mathrm{cm}^{-1}$. The figure illustrates changes in the spectral profile (line shape) as the percentage of whey added to milk was increased in the adulterated samples. Each spectrum corresponds to the average of 3 spectra taken at different spots of the same microdrop sample. The estimated uncertainty of the average spectra was $<5 \%$ for all cases. Two prominent signals can be observed at 1,430 and $1,540 \mathrm{~cm}^{-1}$, which were the most important regions to differentiate distinct levels of fraud. These same Raman shifts were identified by Almeida et al. (2011) as 2 crucial signals for detection of whey in milk powder. Almeida et al. (2011) reported another region (at about $3,000 \mathrm{~cm}^{-1}$ ) that showed differences as the whey concentration increased. Almeida et al. (2012) reported a work with adulteration of milk powder. Different levels of adulteration by adding starch and whey were quantified using Raman spectroscopy and PLS regression. The methodology used by those authors could detect small levels of adulteration: $2.32 \%$ (wt/wt) for added whey and $1.64 \%$ (wt/wt) for added starch.

In the present work, we decided to use ANN, because it has some advantages over traditional multivariate methods used by Almeida et al. (2012). With minor changes, ANN can be trained to perform cluster, classification, or quantitative analysis, eliminating the use of other modeling tools or combinations of multivariate techniques, such as principal component analysis + PLS or principal component analysis + multiple linear regression (MLR), and so on, resulting in time savings and reduction of computational effort and manual labor. Unlike the linear models reported in Almeida et al. (2011, 2012), ANN can be used to model data containing nonlinear relationships with discontinuity or having noise, or, depending on the type of problem, can operate on data with incomplete information. This is especially useful when working with spectroscopy, because not only the Raman spectra but also NIR, UVvisible, and mid-infrared may be affected by several factors inherent to the equipment or the environment (e.g., small variations in the intensity of light or laser, humidity, ambient temperature).

On average, each sample (3 measurements in different spots along the sample) was analyzed in about 5 min. The slowest step was the air-drying step because we lacked an active drying system, such as a convection dryer or hot air oven. No baseline correction, normalization, smoothing, or filtration of Raman spectra was performed. This point deserves special attention, because most of the reported papers on detecting milk adulteration with whey (Almeida et al., 2012), calcium carbonate (Smith et al., 2013), melamine, urea, ammonium sulfate, and dicyandiamide (Cheng et al., 2010; Qin et al., 2013) have performed baseline correction, smoothing, or filtration.

Another point that needs to be emphasized is the possible lack of representativeness of Raman microscopy when applied in food analysis. This is an inherent 


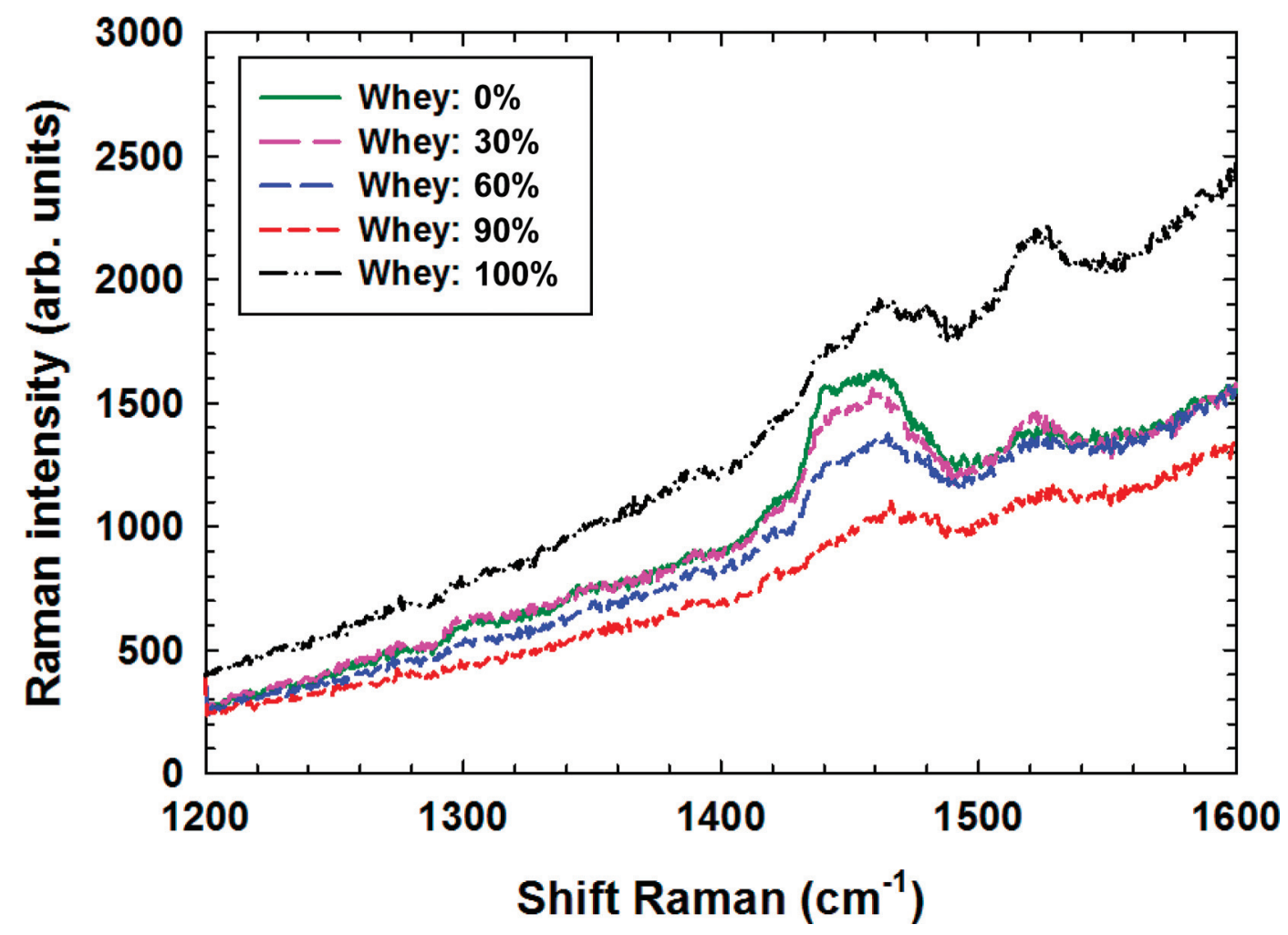

Figure 2. Raman spectra of 5 samples of milk adulterated with whey (from 0 to $100 \%$ ) obtained in the range from 1,200 to $1,600 \mathrm{~cm}^{-1}$. Color version available online.

particularity of the Raman technique and has been debated because most food products are amorphous and anisotropic, and Raman spectra are obtained from very small areas under the sample surface extension, delimited by the focus of the laser. The problem in question can be solved by analyzing as many microscopic fields as possible; in other words, collecting Raman spectra in different spots from the same sample. In this work, Raman measurements were obtained from 3 distinct points of the surface of the 30 samples studied.

Figure 3 exhibits the dispersion between the whey percentage values estimated by the predictor model of PLS (horizontal axis) and the actual values used in sample preparation (vertical axis) with $\mathrm{n}=90$. Even though no clear and well-defined correlation was apparent for adulteration percentage and Raman line shape in Figure 2, regression model adjustments based on linear techniques, such as PLS, presented a reasonable prediction capacity. Figure 3 displays the training and validation data. It presented a suitable fit, with $\mathrm{R}^{2}$ equal to 0.9923 for all data sets. The SIMPLS method for factors extraction and a cross-validation algorithm (both available in SAS JMP Pro) were used for the regression. These 2 parameters were combined to compose a unique score (Eq. [1]), which was used as the criterion for the best predictor model. In this equation, the $t r$ index refers to used data for training network, and vld corresponds to validation data. The best models are those that present the highest score values $(E)$ :

$$
E=\left(\frac{r_{t r}^{2}+r_{v l d}^{2}}{R M S E_{t r}+R M S E_{v l d}}\right) \times 100
$$

Concerning the ANN results, all Raman spectra were acquired in the range from 800 to $1,800 \mathrm{~cm}^{-1}$. However, a smaller range $\left(1,200\right.$ at $\left.1,600 \mathrm{~cm}^{-1}\right)$ was used, because this was the central range, approximately, of all spectra. This approach eliminated the need for a very large number of nodes in the input layer of the neural network, which could make training time too long. The choice of this narrow range was arbitrary and any other range could have been used. Obviously, the adjustment results would be different but, from our experience in adjusting predictive multivariate models, we believe that suitable results would still be obtained.

Table 2 shows that almost all ANN configurations assessed had good prediction capacity, with high values of $r^{2}$ and low values of RMSE for both training and validation data. Thus, ANN can efficiently 


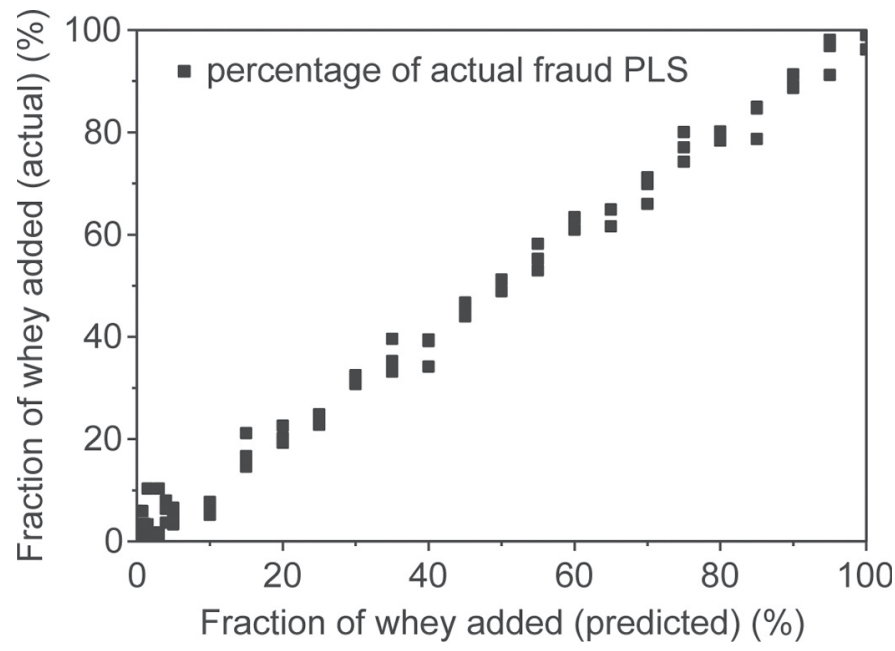

Figure 3. Dispersion values estimated based on current values (known), using a model obtained by partial least squares (PLS) regression; $\mathrm{n}=90$ and $\mathrm{R}^{2}=0.9923$.

extract information contained in Raman spectra, mapping information in the input layer nodes to corresponding values of percentage adulteration in the output layer. Nevertheless, configurations with 8, 9, 13 , and 16 nodes could not "learn" relations treated by those networks. They only "memorized" training data, failing in the prediction of new examples (i.e., used for validation).

Configuration with 19 nodes yielded the highest score. Thus, this model was considered the best predictor. It presented low values of RMSE and high values of $\mathrm{R}^{2}$ (>99.999\%) for both training and validation data, as shown in Figures $4 \mathrm{a}$ and $4 \mathrm{~b}$. Both figures show the dispersion of the values of whey percentage estimated by the neural network (horizontal axis) and the actual values used in the preparation of the samples (vertical axis). Figure 4a refers to data used to train the network, whereas Figure 4b refers to the data used for validation; $\mathrm{R}^{2}=0.9999$ in both figures. When working with neural networks, a part of the experimental data is reserved, a priori, to fit the predictive model. These are the data for network training. After the training phase, the fitted model is checked with the validation data, also reserved a priori. Validation data, as the name suggests, are data that have never been tested previously with the predictive model provided by the neural network. They are used retrospectively to evaluate and verify that the model obtained is accurate in prediction and able to correctly predict values previously "unknown" by ANN. In our experiments, the network was able to correctly predict all the values used for validation, and so the model was considered satisfactory. Simpler configurations, as that with 15 hidden nodes, also had high prediction capacity, superior to that obtained by PLS.

In practice, levels of adulteration by the intentional addition of whey to milk rarely exceed 25 to $30 \%$. In the present work, we extended the percentage adulteration up to $100 \%$ to assess how the methodology behaved throughout the full range of possible levels. The responses obtained were homogeneous over the studied range. Moreover, no preferred range or range of greater or lesser sensitivity was identified in the responses. Therefore, the method is equally effective for any level of adulteration. The method showed ruggedness, linearity, and high capacity of measurement.

Table 2. Coefficient of determination $\left(\mathrm{R}^{2}\right)$ values and root mean square error (RMSE) between actual values (known) and predicted for the percentage of fraud in milk by addition of serum

\begin{tabular}{|c|c|c|c|c|c|}
\hline \multirow{2}{*}{$\begin{array}{l}\text { Hidden nodes }{ }^{1} \\
\text { (no.) }\end{array}$} & \multicolumn{2}{|c|}{ Training } & \multicolumn{2}{|c|}{ Validation } & \multirow[b]{2}{*}{ Score } \\
\hline & $\mathrm{R}^{2}$ & RMSE & $\mathrm{R}^{2}$ & RMSE & \\
\hline 5 & 0.9208 & 9.4898 & 0.9875 & 3.6608 & 14.51 \\
\hline 6 & 0.9701 & 5.8432 & 0.9821 & 4.2436 & 19.35 \\
\hline 7 & 0.9910 & 3.1600 & 0.9974 & 1.8467 & 39.71 \\
\hline 8 & 0.9999 & 0.0773 & 0.6253 & 20.3805 & 7.94 \\
\hline 9 & 0.9939 & 2.6152 & 0.3167 & 24.8374 & 4.77 \\
\hline 10 & 0.8990 & 10.7322 & 0.9536 & 6.8664 & 10.53 \\
\hline 11 & 0.9884 & 3.6718 & 0.9998 & 0.3607 & 49.30 \\
\hline 12 & 0.9999 & 0.2908 & 0.8241 & 10.5490 & 16.83 \\
\hline 13 & 0.9999 & 0.0269 & 0.0742 & 32.9915 & 3.25 \\
\hline 14 & 0.9984 & 1.3475 & 0.9750 & 5.6480 & 28.21 \\
\hline 15 & 0.9985 & 1.2859 & 0.9999 & 0.2583 & 129.41 \\
\hline 16 & 0.9999 & 0.0223 & 0.6834 & 19.2072 & 8.75 \\
\hline 17 & 0.9926 & 2.8582 & 0.9964 & 2.1890 & 39.41 \\
\hline 18 & 0.8153 & 14.7540 & 0.9999 & 0.1254 & 12.20 \\
\hline 19 & 0.9999 & 0.0629 & 0.9999 & 0.0469 & 1821.31 \\
\hline 20 & 0.9935 & 2.6639 & 0.9999 & 0.3863 & 65.35 \\
\hline
\end{tabular}

${ }^{1}$ Neural networks were evaluated with settings between 5 and 20 nodes in the hidden layer. 


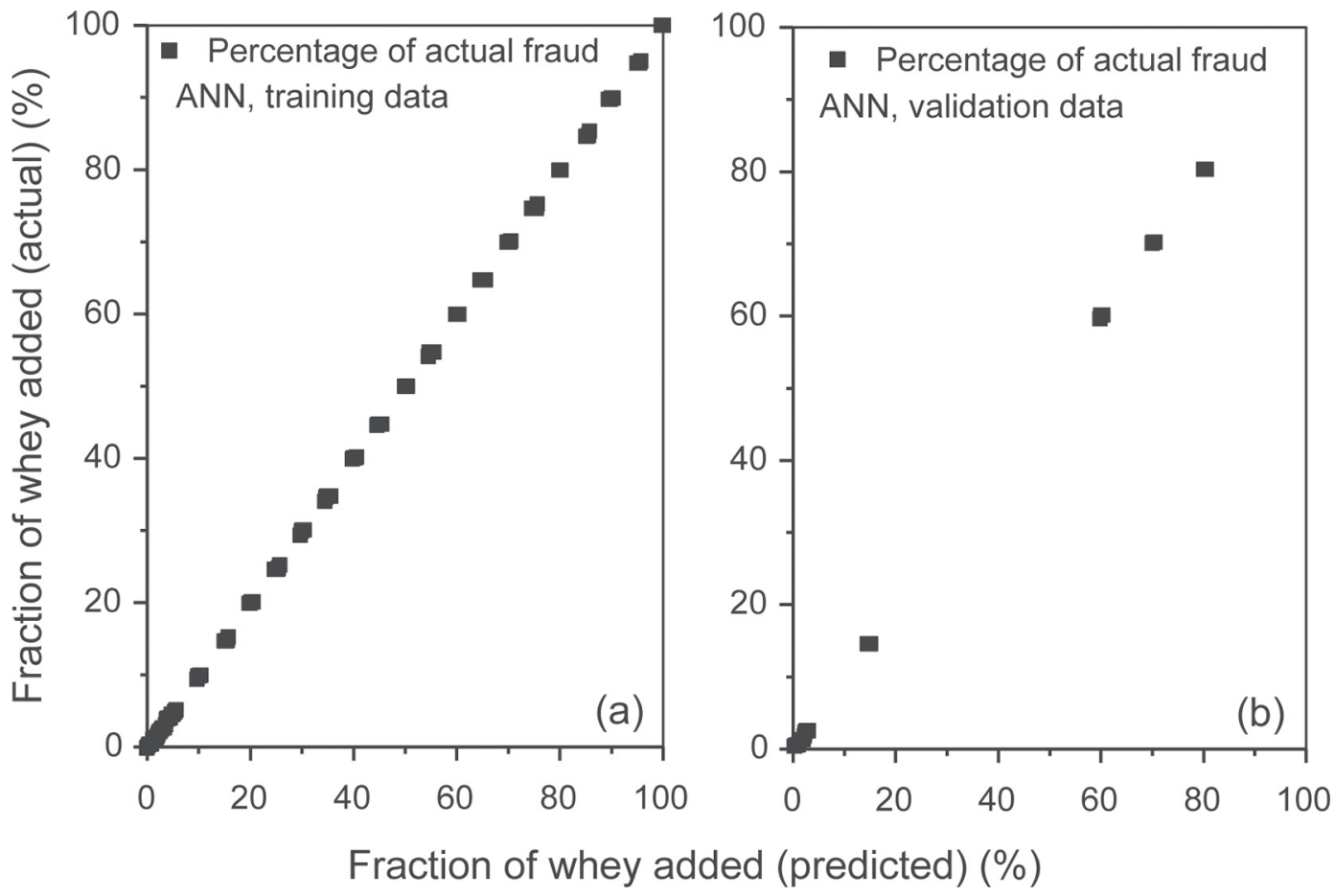

Figure 4. Comparison between the fraction of whey added (actual) and the one predicted by a model obtained by artificial neural networks: (a) training data; and (b) validation data; $\mathrm{n}=90$, hidden nodes $=19$, and $\mathrm{R}^{2}=0.9999$.

Comparison of the proposed methodology with other well-established techniques such as ELISA and HPLC is also important. A Raman analysis takes about $1 \mathrm{~min}$ and is therefore faster than the HPLC analysis, which takes, on average, 15 to $30 \mathrm{~min}$. Additionally, ELISA is a time-consuming analysis, because it requires an incubation time of about 30 to $60 \mathrm{~min}$ to allow chemical reactions to occur so that the absorbance of the samples can be read in a microplate spectrophotometer. Additionally, the total number of steps involved in the operation and use of Raman is fewer than the total number of steps required to perform an HPLC analysis. The development of an analytical method for Raman is much simpler than for an analytical method for HPLC, because it involves only the proper focus of the sample under the lens of an optical microscope, adjustment of the laser wavelength, and cooling the CCD with liquid nitrogen to $-123.0^{\circ} \mathrm{C}$. Another advantage of the Raman method compared with HPLC is that Raman analysis is made on a wide range of Raman shifts, so all vibrational information from a large number of chemical groups and compounds in various structures present in the sample is considered. The analysis of HPLC, unlike Raman, is based on less information, and separation efficiency (and quality of results) will depend on the method used and the skill and knowledge of the analyst. Moreover, different models of portable
Raman spectrometers are available for in situ analysis. Although portable HPLC can also be found, the Raman method does not require sample or buffer preparation, or use of preparation and guard columns.

With respect to industrial models and pilot-scale models based on multivariate linear PLS, calibration can become relatively difficult, especially when it is necessary to process many discrete points or values taken from a spectrum. It makes it difficult or infeasible to use many discrete points in the construction of automatic control systems and portable embedded modules, which can be used directly in the plant for control purposes and standardization of production processes. Neural networks can work around this problem and handle it in a simpler way, generating prediction models that are robust and capable of being stored within a single microchip.

We believe the proposed methodology can be extended to other dairy products. Typically, the spectra obtained by the Raman microscope correspond to a unique and unambiguous fingerprint of the food under study, whereas ANN comprises a set of algorithms that can be used to sort, map, and quantify the components of food, based on the data contained in this fingerprint. Recently, we applied these techniques to mixtures of olive oil and soybean oil and achieved excellent results (Mendes et al., 2015). 


\section{CONCLUSIONS}

The use of confocal Raman microscopy together with ANN is an excellent method to quantify adulteration of milk by whey because of its high speed, simplicity, and efficiency. Raman microscopic analyses required only environmental air-drying of the samples before measurement. Moreover, drying time was $2 \mathrm{~h}$, which could be reduced to a few minutes by using a dryer. After data acquisition, no filtration, baseline correction, normalization, or smoothing procedure was applied to the spectra; only some high-energy spikes were removed. Therefore, this method constitutes a promising method for food inspection, sanitary vigilance, and safety control. A high-capacity prediction model was obtained using $\mathrm{ANN}$, with $\mathrm{R}^{2}$ of 0.9999 . Alternatively, ANN can be replaced by a linear model adjusted using PLS, which also exhibited reasonable results for the prediction of percentage of whey added $\left(\mathrm{R}^{2}=0.99\right)$. The combined use of Raman and ANN could be used in several other foods and applications.

\section{ACKNOWLEDGMENTS}

The authors thank the Brazilian agencies CAPES (Coordenação de Aperfeiçoamento de Pessoal de Nível Superior, Brasília, Brazil), FAPEMIG (Fundação de Amparo à Pesquisa do Estado de Minas Gerais, Belo Horizonte, Brazil), and CNPQ (Conselho Nacional de Desenvolvimento Científico e Tecnológico, Brasília, Brazil) for the financial support. We also express our thanks to the Faculty of Pharmacy of the Federal University of Juiz de Fora (Brazil) and SAS Institute Inc. (subsidiary in São Paulo, Brazil) for allowing the use of the statistical package SAS JMP.

\section{REFERENCES}

Almeida, M. R., K. S. Oliveira, R. Stephani, and C. L. F. Oliveira 2011. Fourier-transform Raman analysis of milk powder: A potential method for rapid quality screening. J. Raman Spectrosc. 42:1548-1552.

Almeida, M. R., K. S. Oliveira, R. Stephani, and C. L. F. Oliveira. 2012. Application of FT-Raman spectroscopy and chemometric analysis for determination of adulteration in milk powder. Anal. Lett. 45:2589-2602.

Baughman, D. R., and Y. A. Liu. 1995. Neural Networks in Bioprocessing and Chemical Engineering. Academic Press, San Diego, CA.

Borin, A., M. F. Ferrão, C. Mello, D. A. Maretto, and R. J. Poppi. 2006. Least-squares support vector machines and near infrared spectroscopy for quantification of common adulterants in powdered milk. Anal. Chim. Acta 579:25-32.

Brazil. 2006. Normative Instruction 68, 12 Dec. Formalizes the official analytical physical-chemical methods for milk and dairy products control, in accordance to the annex from this Normative Instruction. Ministry of Agriculture, Livestock and Food Supply (MAPA), Brasília, Brazil.
Chakraborty, R., and H. Sahu. 2014. Intensification of biodiesel production from waste goat tallow using infrared radiation: Process evaluation through response surface methodology and artificial neural network. Appl. Energy 114:827-836.

Chávez, N. A., J. Jauregui, L. A. Palomares, K. E. Macías, M. Jiménez, and E. Salinas. 2012. A highly sensitive sandwich ELISA for the determination of glycomacropeptide to detect liquid whey in raw milk. Dairy Sci. Technol. 92:121-132.

Chávez, N. A.. E. Salinas, J. Jauregui, L. A. Palomares, and K. Macias. 2008. Detection of bovine milk adulterated with cheese whey by Western blot immunoassay. Food Agric. Immunol. 19:265-272.

Cheng, Y., Y. Dong, J. Wu, X. Yang, H. Bai, H. Zheng, D. Ren, Y. Zou, and M. Li. 2010. Screening melamine adulterant in milk powder with laser Raman spectrometry. J. Food Compos. Anal. 23:199-202.

Dieing, T., and O. Hollricher. 2008. High-resolution, high-speed confocal Raman imaging. Vib. Spectrosc. 48:22-27.

Galindo-Amaya, L. L., E. Valbuena-Colmenares, and E. Rojas-Villarroel. 2006. Standardization of glycomacropeptide detection with SDS-PAGE as a milk adulteration index. Rev. Científ. (Maracaibo) $16: 308-314$

Giovannozzi, A. M., F. Rolle, M. Sega, M. C. Abete, D. Marchis, and A. M. Rossi. 2014. Rapid and sensitive detection of melamine in milk with gold nanoparticles by surface enhanced Raman scattering. Food Chem. 159:250-256.

Haykin, S. 1999. Neural Networks-A Comprehensive Foundation. 2nd ed. Prentice Hall International Inc., Upper Saddle River, NJ.

Jawaid, S., F. N. Talpur, S. T. H. Sherazi, S. M. Nizamani, and A. A. Khaskheli. 2013. Rapid detection of melamine adulteration in dairy milk by SB-ATR-Fourier transform infrared spectroscopy. Food Chem. 141:3066-3071.

Kapila, R., P. K. Kavadi, and S. Kapila. 2013. Comparative evaluation of allergic sensitization to milk proteins of cow, buffalo and goat. Small Rumin. Res. 112:191-198.

Kawakami, H., Y. Kawasaki, S. Dosako, M. Tanimoto, and I. Nakajima. 1992. Determination of kappa-casein glycomacropeptide by high performance liquid chromatography without trichloroacetic acid pretreatment. Milchwissenschaft 47:688-693.

Koning, P., J. Elisses, and H. De Vries. 1966. A method for the detection of small percentages of whey powder in milk powder. Neth. Milk Dairy J. 20:203-212.

López-López, M., and C. García-Ruiz. 2014. Infrared and Raman spectroscopy techniques applied to identication of explosives. Trends Analyt. Chem. 54:36-44.

McCreery, R. L. 2000. Raman Spectroscopy for Chemical Analysis. Monographs of Analytical Chemistry and its Applications. Vol. 157. Wiley-Interscience, Columbus, OH.

Mendes, T. O., R. A. Rocha, B. L. S. Porto, M. A. L. Oliveira, V. Anjos, and M. J. V. Bell. 2015. Quantification of extra-virgin olive oil adulteration with soybean oil: A comparative study of NIR, MIR, and Raman spectroscopy associated with chemometric approaches. Food Analyt. Methods http://dx.doi.org/10.1007/ s12161-015-0121-y.

Neelima, R. Sharma, and Y. S. Rajput. 2012. Direct estimation of sialic acid in milk and milk products by fluorimetry and its application in detection of sweet whey adulteration in milk. J. Dairy Res. 79:495-501.

Neelima, R. Sharma, Y. S. Rajput, and B. Mann. 2013. Chemical and functional properties of glycomacropeptide (GMP) and its role in the detection of cheese whey adulteration in milk: A review. J. Dairy Sci. Technol. 93:21-43.

Olieman, C., and J. Bedem. 1983. A sensitive HPLC method of detecting and estimating rennet whey total solids in skim milk powder. Neth. Milk Dairy J. 37:27-36.

Qin, J., K. Chao, and M. S. Jim. 2013. Simultaneous detection of multiple adulterants in dry milk using macro-scale Raman chemical imaging. Food Chem. 138:998-1007.

Ramji, S., G. Latha, and S. Ramakrishnan. 2009. Estimation and interpolation of underwater low frequency ambient noise spectrum using artificial neural networks. Appl. Acoust. 70:1111-1115. 
Recio, I., M. R. García-Risco, R. López-Fandiño, A. Olano, and M. Ramos. 2000. Detection of rennet whey solids in UHT milk by capillary electrophoresis. Int. Dairy J. 10:333-338.

Santos, P. M., E. R. Pereira-Filho, and L. E. Rodrigues-Saona. 2013. Rapid detection and quantification of milk adulteration using infrared microspectroscopy and chemometrics analysis. Food Chem. 138:19-24.

Smith, G. P. S., K. C. Gordona, and S. E. Holroydb. 2013. Raman spectroscopic quantification of calcium carbonate in spiked milk powder samples. Vib. Spectrosc. 67:87-91.

Sorhauge, T., and L. Stepaniak. 1997. Psychrotrophs and their enzymes in milk and dairy products: Quality aspects. Trends Food Sci. Technol. 8:35-41.

Unluturk, S., M. Pelvan, and M. S. Unluturk. 2013. The discrimination of raw and UHT milk samples contaminated with penicillin $\mathrm{G}$ and ampicillin using image processing neural network and biocrystallization methods. J. Food Compos. Anal. 32:12-19.
Vilela, S. C. 1987. Detection de suero de queseria agragado a leche pasteurizada y leche em polvo, por determinacion del glicomacropeptídeo por eletroforesis. MS Thesis. Universidad Austral de Chile, Valdívia, Chile.

Warren, L. 1959. The thiobarbituric acid assay of sialic acids. J. Biol. Chem. 234:1971-1975.

Wolfschoon-Pombo, A. F., and A. P. E. F. Pinto. 1985. A qualitative method for the detection of rennet whey in milk. Cienc. Tecnol. Aliment. 5:111-115.

Zhang, L. G., X. Zhang, L. J. Ni, Z. B. Xue, X. Gu, and S. X. Huang. 2014. Rapid identification of adulterated cow milk by non-linear pattern recognition methods based on near infrared spectroscopy. Food Chem. 145:342-348.

Zheng, H., K. C. Gordon, and D. W. Everett. 2013. Innovative application of confocal Raman microscopy to investigate the interaction between trans-2-hexenal and bovine milk fat globules. Int. Dairy J. $32: 68-70$ 